\title{
51. Jahrestagung der ASCO Illumination \& Innovation - wohin die Reise geht
}

Chicago. Die weltweit größte Zusammenkunft der Onkologen fand in diesem Jahr zwischen dem 29. Mai und 2. Juni unter dem Leitmotiv 'Illumination \& Innovation: Transforming Data into Learning' statt. Für den Präsidenten der 51. Jahrestagung der ASCO (American Society of Clinical Oncology), Dr. Peter P. Yu/ Palo Alto Medical Foundation, war die Wahl dieses Leitmotivs angesichts der rekordverdächtigen Anzahl der ca. 6.000 eingereichten Abstracts, naheliegend: Verglichen zum Vorjahr sei ein Anstieg um 7,5\% zu verzeichnen - eine regelrechte Datenexplosion, die möglichst effektiv dazu genutzt werden müsse, um Krebspatienten den schnellstmöglichen Zugang $\mathrm{zu}$ innovativen onkologischen Therapieoptionen zu verschaffen.

\section{Individualisierung durch Vernetzung}

Als einer der Vordenker für digitale Informationstechnologien im Gesundheitswesen plädierte Yu für den lernenden Umgang mit Daten-Ressourcen ('big health data'), die auf eine qualitative und Outcome-basierte Verbesserung der onkologischen Versorgung abziele und neue Versorgungsmodelle generiere, um in allen Lebensbereichen eine Verbesserung der Krebstherapie anzustreben. Die ASCO initiierte dazu auch die Entwicklung einer HealthIT-basierten Plattform (CancerLinQ), die sich der nationalen Vernetzung von Patienten- und Therapiedaten für die Forschung bzw. Versorgung widmen und Onkologen in Zukunft eine patientenindividuelle Behandlung erleichtern soll, die sich an der aktuellen Evidenz und Guidelines orientiert (cancerlinq.org).

\section{Neue Hoffnung für «Hard-to-treat»-Tumore?}

$\mathrm{Zu}$ den beherrschenden Hot Topics aus der aktuellen Therapieforschung gehörten auch in diesem Jahr wieder neue Daten zur immunvermittelten Tumortherapie, die zunehmend auch bei Tumortypen untersucht werden, die historisch als weniger immunogen galten: Folgerichtig sorgten Vorträge, Educational Sessions oder Posterpräsentationen rund um den Einsatz immunonkologisch aktiver Substanzen für den lebhaftesten Zulauf während der Kongresstage. Zum Stellenwert der Immuntherapien bei bislang nur schwer therapiebaren Tumorentitäten richtete die ASCO sogar eine eigene Pressekonferenz aus. Wie Dr. Lynn Schuchter betonte, seien die Zeiten vorbei, in den der immunonkologische Behandlungsansatz vor allem als Durchbruchinnovation beim malignen Melanom angesehen wurde. Die onkologische Fachwelt nehme derzeit mit Staunen zu Kenntnis, dass die neue Therapiemodalität auch noch bei refraktären Tumortypen wirksam ist, bei denen alle anderen Therapien bereits versagt hätten. Wie Dr. Gregory A. Masters erinnerte, sei man noch vor 5 Jahren davon ausgegangen, dass es beim Lungenkarzinom unmöglich sei, ein immunvermitteltes Therapieansprechen zu erreichen. Die Ergebnisse der randomisierten Phase-III-Studien seien 'game changing' und würden dafür sprechen, dass der bisherige Therapiestandard in Zweitlinie beim fortgeschrittenen, nicht-squamösen nicht-kleinzelligen Lungenkarzinoms (NSCLC) durch Nivolumab abgelöst würde. Als LateBreaking-Abstracts wurden vor diesem Hintergrund erste, vielversprechende Phase-I/II-Daten beim kolorektalen Karzinom, KopfHals-Tumoren sowie beim fortgeschrittenen Leberzellkarzinom bzw. Phase-III-Daten in der Zweitlinientherapie des nicht-squamösen NSCLC besprochen.

Erstmals wurden Phase-III-Daten zur PD-1-Inhibitortherapie (Nivolumab, $\mathrm{n}=292$ ) versus Docetaxel $(\mathrm{n}=290)$ beim nicht-squamösen NSCLC vorgestellt, die den Überlebensvorteil bei weniger Nebenwirkungen nachwiesen (J Clin Oncol 2015;33suppl; abstr LBA109).

Einer frühen Dosiseskalationsstudie zufolge, die bei Patienten ( $n=47)$ mit einem hepatozellulären Karzinom (HCC) durchgeführt wurde, erreichten vorbehandelte (mindestens eine systemische Vortherapie einschließlich Sorafenib) HCC-Patienten unter der PD-1-Inhibitortherapie (Nivolumab) eine Gesamtüberlebensrate von 62\% nach einem Jahr (J Clin Oncol 2015;33suppl; abstr LBA101). In der vorliegenden Studie waren sowohl HBV- als auch $\mathrm{HCV}$-infizierte Patienten eingeschlossen. Interessanterweise ist aus früheren Untersuchungen über das PD-1-Molekül bekannt, dass es in der Leber antivirale Immunreaktionen unterdrücken kann (Iwai et al., J Exp 2003).

Bei Patienten mit einem progredienten und metastasierten, therapierefraktären kolorektalen Karzinom (CRR, $n=25)$, waren Mismatch-Repair-(MMR-)defiziente Tumore mit einem Tumorschrumpfen unter der PD-1-Inhibitortherapie (Pembrolizumab) assoziiert, wohingegen MMR-profiziente Tumore kein Ansprechen entwickelten (J Clin Oncol 2015;33suppl; abstr LBA100).

\section{KARGER}

Fax +497614520714 (c) 2015 S. Karger GmbH, Freiburg

$2296-5270 / 15 / 0388-0386 \$ 39.50 / 0$ 


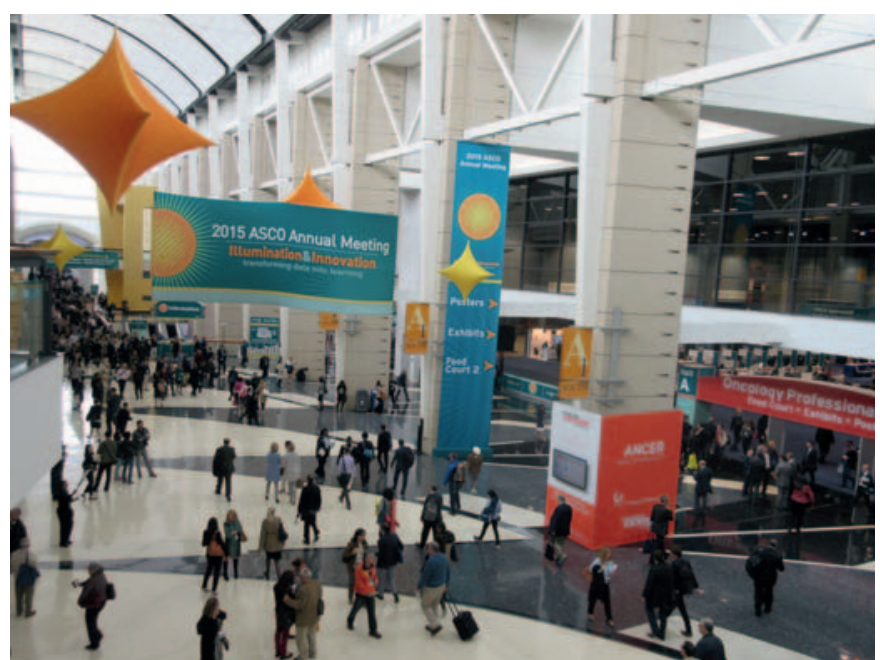

Das Prinzip der PD-1-Blockade (Pembrolizumab) könnte auch für therapierefraktäre oder metastasierte Kopf-Hals-Tumore eine neue Therapieoption bieten: Ein Anteil von 57\% der teilweise vorbehandelten Patienten mit einem Plattenepithelkarzinom im Kopf-/Hals-Bereich erfuhr eine Schrumpfung der Tumorgröße (J Clin Oncol 2015;33suppl; abstr LBA6008).

\section{Paradigmenwechsel in der Krebstherapie: Immunsystem als Therapietarget}

Das adaptive Immunsystem bringe gleich drei Voraussetzungen mit, um Tumorzellen nachhaltig in Schach zu halten, erklärte der diesjährige Preisträger der ASCO Science Oncology Awards, Prof. James P. Allison, University of Texas, Houston: die ausgeprägte Antigenspezifität, die Anpassungsfähigkeit sowie das immunologische Gedächtnis (T-Gedächtniszellen). Auf der Suche nach Fremdantigenen sei es für T-Lymphozyten letztlich egal, ob es sich z.B. um einen viralen Eindringling oder eine entartete körpereigene Zelle jedweden Organs handeln würde. Allison und seine Mitarbeiter hatten in den 1990er Jahren erstmals die Bedeutung von Rezeptormolekülen erkannt, die eine Schlüsselfunktion bei der Steuerung der T-Zell-Aktivierung und -Proliferation spielten, allen voran das inzwischen am besten charakterisierte Rezeptorprotein CTLA-4. Als bahnbrechend sollte sich der Ansatz erweisen, durch eine therapeutische Antikörperblockade die negative T-Zell-Regu- lation aufzuheben und über die Brechung der peripheren Toleranz eine anhaltende Immunreaktion hervorzurufen, die sich sogar gegen etablierte Tumore richten würde (Leach DR et al., Science 1996;271:1734-1736). Hervorgegangen war daraus der erste Immun-Checkpoint-Inhibitor Ipilimumab, der heute beim metastasierten malignen Melanom eingesetzt wird und Langzeitremissionen induzieren kann.

Die Entdeckung, dass es neben dem CTLA-4-Molekül weitere Rezeptorproteine gibt, die zur Inaktivierung von T-Zellen führen, führte auch zur Entwicklung eines weiteren Immun-CheckpointInhibitors, der sich gegen den PD-1-Rezeptor (programmed death 1, PD-1) richtet. Die Familie der Immun-Checkpoint-Moleküle umfasst daneben viele weitere Rezeptorproteine, die für eine künftige therapeutische Intervention in Frage kommen: Viele Tumore können gleich mehrere immunregulative Signalwege co-optieren, um Immunabwehrreaktionen zu dämpfen, die dem Tumorwachstum entgegenstehen könnten. Dass die Kombination von ImmunCheckpoint-Inhibitoren mit distinkten Wirkmechanismen zu einer additiven oder synergistischen Verstärkung mit einer weiteren Verbesserung der Gesamtüberlebensraten führen kann, legen z.B. die Daten aus einer ersten klinischen Kombinationsstudie (Ipilimumab plus Nivolumab) nahe, die beim malignen Melanom vorgestellt wurde (ASCO 2015, \#LBA1). Die ersten Hinweise auf eine Potenzierung der antitumoralen Aktivität über die kombinierte Immun-Checkpoint-Blockade stammten aus einem Mausmodell beim Adenomkarzinom des Kolon (Korman A et al., J Immunol 2007;178:S82).

Die Idee, das Immunsystem und nicht mehr die einzelne Tumorzelle als Therapieziel zu bearbeiten, hätten viele Onkologen seinerzeit für ein aussichtsloses Unternehmen gehalten, erinnerte sich Allison bei seiner Preisträgerrede. Inzwischen, resümierte Allison, könnten mehr und mehr Patienten damit rechnen, unter den erweiterten Möglichkeiten der Checkpoint-Blockade ein über Jahre währendes Überleben zu erreichen, während es früher lediglich um die Verlängerung des medianen Überlebens um wenige Monate gegangen sei.

Dr. med. Yuri Sankawa, Stuttgart

Quellen

ASCO-Pressekonferenz 'New Immunotherapies for Multiple Hard-to-treat Cancers', 29.5.2015 und 'Plenary Session Including the Science of Oncology Award and Lecture' am 31.5.2015. 